\title{
INTERSTITIAL BRACHYTHERAPY IN THE MANAGEMENT OF UTERINE CERVICAL CANCER- SINGLE INSTITUTIONAL STUDY
}

\author{
Thirukurungudi Narayanan Vijayasree ${ }^{1}$, Subramanian Saravanan ${ }^{2}$
}

1Associate Professor, Department of Radiotherapy, Government Royapettah Hospital and Kilpauk Medical College, Chennai, Tamilnadu, India.

2Professor, Department of Radiotherapy, Government Royapettah Hospital and Kilpauk Medical College, Chennai, Tamilnadu, India. ABSTRACT

\section{BACKGROUND}

The objective of this study is to report the clinical outcome of patients with carcinoma cervix treated by radiation therapy including brachytherapy (BT), using template based Interstitial Brachytherapy (ISBT), in whom conventional Intracavitary Application (ICA) is not possible and to determine the local control and the acute and late radiation induced toxicities.

\section{MATERIALS AND METHODS}

From Apr 2016 to Mar 2018, among histologically proven cervical cancer patients, 30-70 yrs. of age, stages from IB to IIIB, completed concurrent chemo-radiation (radical/adjuvant) and referred for High Dose Rate Brachytherapy (HDR BT), thirty patients, in whom conventional ICA is not possible are treated using template based, image guided ISBT. The dose prescribed per fraction of HDR-ISBT in our institute varied from 3-4 Gy/fraction to a total of 12-21 Gy in 4-6 fractions. D90 for HR-CTV of BT, cumulative EQD2 of HR-CTV and the cumulative D2cc for the bladder, rectum, and sigmoid, (Combined EBRT and BT) were analysed. Patients were evaluated for disease related parameters, treatment-related toxicities and tumour-response rates at 6 weeks, then every month for first 3 months and every 3 months thereafter. MRI is done at end of 3 months.

\section{RESULTS}

Among 735 patients referred for brachytherapy, 30 patients, who are not fit for conventional ICA, are treated by HDR ISBT.19 patients were using Syed-Neblett and 11 using Mupitz template. Median number of needles used was 15. The median BT Dose planned was 23.6 Gy and median HR-CTV D90 was 17.5 Gy. The median cumulative EQD2 for HR-CTV, D2cc for the bladder, rectum, and sigmoid were 67.5, 62.6, 64.36, 54.83 Gy respectively. The median V100 for HR-CTV was 70 ml. Local control (LC) rate at first follow up ( 3 months) is $43.3 \%$ and at $1 \mathrm{yr}$. is $41.38 \%$. The median Progression-free survival time for two year follow up was 19.92 months. One patient (3.33\%) had distant metastasis, two patients $(6.67 \%)$ experienced grade 2 rectal bleeding but none had bladder complications.

\section{CONCLUSION}

Treatment by image guided ISBT for carcinoma cervix patients in whom conventional application is not possible, results in good dose-volume histogram values and favourable local control with acceptable toxicities, is depicted through this prospective single institutional study with a lesser number of patients.

\section{KEY WORDS}

Carcinoma Cervix, Interstitial Brachytherapy.

HOW TO CITE THIS ARTICLE: Vijayasree TN, Saravanan S. Interstitial brachytherapy in the management of uterine cervical cancer- single institutional study. J. Evolution Med. Dent. Sci. 2018;7(50):5368-5372, DOI: 10.14260/jemds/2018/1188

\section{BACKGROUND}

In countries like India, carcinoma cervix remains the leading cause of cancer related deaths and is treated mainly with concurrent chemo radiation. Brachytherapy (BT) plays an important role because it has intrinsic property of steep dose gradient, by delivering relatively higher dose to target, and a much lower dose to the organs at risk.[1,2]Tumours having smaller volume, which lie within the isodose line generated by conventional intracavitary brachytherapy (ICBT), using

'Financial or Other Competing Interest': None.

Submission 02-11-2018, Peer Review 26-11-2018,

Acceptance 03-12-2018, Published 10-12-2018.

Corresponding Author:

Thirukurungudi Narayanan Vijayasree,

No. 5/9, $5^{\text {th }}$ Main Road,

Ellaiamman Nagar,

Korattur, Chennai,

Tamilnadu, India.

E-mail:tnvijayasree@gmail.com

DOI: $10.14260 /$ jemds/2018/1188 intrauterine tandem and vaginal (Ovoid/Ring) sources, calculated based on the Manchester principles,[3,4] can be treated optimally. Tumours, which are slightly larger than isodose line created by ICBT, can be treated by image-guided adaptive brachytherapy (IGABT), $[5,6,7,8]$ with or without additional interstitial needle placement (Hybrid Brachytherapy - HBT).[9,10,11,12] But, as per American Brachytherapy Society (ABS) Guidelines, high dose rate (HDR) interstitial brachytherapy (ISBT) is a better option, for patients with carcinoma cervix in certain clinical scenarios like bulky lesions, a narrow vaginal apex, inability to negotiate the cervical os, extensive disease up to the lateral parametria or pelvic sidewall, and involvement of lower third of vagina, in an attempt to improve local control. This technique aims to tailor the dose of irradiation to the anatomy of the patient with a better target volume coverage. In this study, our Institution being the only Government Institution practicing ISBT in our state, we can select cases for ISBT, in an attempt to improve local control. 


\section{Purpose}

Uterine cervical cancer patients referred to our department for Brachytherapy, after completion of EBRT of $50 \mathrm{~Gy} / 25 \#$, in 200 cGy/\# along with weekly Cisplatin and those who were not the candidates for conventional ICA are recruited. They are treated by HDR-ISBT to get a better dose to the target. Two main perineal templates types described in the literature, the Martinez Universal Perineal Interstitial Template (MUPIT) ${ }^{[13]}$ and the Syed-Neblett template $[$ were used depending upon the disease extension at the time of BT.

\section{Objective}

The objective of this study is to report the clinical outcome of carcinoma cervix patients treated by Radiation therapy including brachytherapy (BT), using Template based interstitial brachytherapy (ISBT), in whom conventional Intracavitary application (ICA) is not possible and to determine the local control and the acute and late radiation induced toxicities.

\section{Study Design}

Prospective longitudinal observational study.

\section{MATERIALS AND METHODS}

From April 2016 to March 2018, uterine cervical cancer patients, with 30-70 yrs. of age, histologically proven cervical squamous, adeno or adenosquamous carcinoma, FIGO stages from IB to IIIB and referred for brachytherapy are enrolled. All of received concurrent chemo radiation with $40 \mathrm{mg} / \mathrm{m} 2$ of cisplatin every week and EBRT, delivered by 3D conformal radiotherapy with $15 \mathrm{MV}$ linear accelerator (Clinac®iX, Varian Medical System). Patients are then evaluated for Conventional ICBT using tandem and ovoids/ Central vaginal cylinder (CVC). Patients who are not suitable for conventional ICBT/CVC or in whom conventional ICBT is unlikely to cover the entire tumour are recruited and treated with interstitial brachytherapy using MUPIT/SYED applicator, as per ABS guidelines. Patients with Haemoglobin minimum $10 \mathrm{gm} \%$, performance status $70 \%$ or more (Karnofsky scale) or $0-2$ (ECOG) are only eligible. Patients with distant metastasis are excluded from this study. HDR-ISBT was delivered using 192Ir remote after loading system (Micros electron HDRV3, Nucletron, supplied by Elekta). Computed tomography (CT)based Image-guided HDR-ISBT is done. Pre-Implant planning done and the position for needle placement in the template is selected by doing per vaginal examination to detect lateral, anterior and posterior extension of tumour. The depth to which the needles must be inserted is decided depending on the superior and inferior limits. The cylinder length in the vagina is chosen according to per vaginal examination. Preimplant anaesthetic fitness obtained, and bowel preparation done the day before the implant, since the Implant may be kept in situ for 2 to 3 days. The patient in the lithotomy position and implantation procedure is performed under spinal anaesthesia, Fig. 1. In brief, bladder catheterized, Perineal template placed, and needles were inserted. Whenever possible, a central tandem was also inserted. The template is sutured to the perineum, cover plate is placed over the template and screws tied to prevent the needles from displacement. Sterile gauze is placed between the template and the skin. A rectal tube is inserted. CT Simulation was carried out using the SOMATOM Definition AS 20 wide- bore open model Siemens Simulator, to get CT images with slice thickness of $2 \mathrm{~mm}$. The image-guided brachytherapy planning was performed on those images. Treatment planning was done with a brachytherapy planning system (Oncentra® TPS V 4.3) Fig. 2 and Treatment carried out by Nucletron-microSelectron - 18 channel HDR Brachytherapy Unit.

In the CT images, clinical target volume (CTV) that encompassed the volume occupied by the tumour, and also OAR, including the bladder, rectum, and sigmoid, are contoured. Information obtained from the per vaginal examination during the procedure, and CT/ magnetic resonance images (MRIs), which were taken before BT in the majority of cases, were taken into account for determining CTV. Dose calculation is performed by geometrical optimization or dose point volume optimization, followed by fine manual graphical modification to cover the CTV with the prescription dose while keeping the dose to OAR within allowable limits. We were keen during planning that, Hyperdose sleeve, which represents $200 \%$ of the prescribed dose of a particular needle, should not fuse to that of another which will increase the high dose volume. The prescription dose per fraction of HDR-ISBT in our institute varied from 3-4 Gy/\# to a total of 12-21 Gy in 4-6\#. The dose constraint of the cumulative dose of WPRT and HDR-ISBT for the rectum and the bladder D2cc is 75 Gy and 90 Gy (EQD2, $\alpha / \beta=3$ Gy), respectively.

When computing the total dose of EBRT and HDR-ISBT, the equivalent dose in 2 Gy fractions (EQD2) is calculated, according to the linear-quadratic dose-effect model.[14,15,16] Tumour and OAR doses are calculated assuming $\alpha / \beta$ as $10 \mathrm{~Gy}$ and $3 \mathrm{~Gy}$, respectively. The minimum dose covering $90 \%$ of the CTV (CTV D90) in EQD2 is taken as the representative dose of HDR-ISBT.

In the entire treatment period of 3-4 days, needle placement was done once, and patients were treated twice daily at an interval of 6 hours. The patients were admitted in hospital during the treatment period (3-4 days) and treated with adequate antibiotic coverage and proper hydration. The implant was removed after completion of treatment.

\section{Post-Implant Follow-Up}

All patients were advised to come for follow up every month for the first six months and once in 3 months up to two years. During follow-up, patients were evaluated for local response, complications and distant metastasis.

Demographic, disease-specific characteristics, treatment details, and outcome data are analysed.

Complete remission (CR) was defined as no evidence of disease 3 months after the end of treatment, evaluated by clinical examination and MRI. The calculation of local control rate (LC) was done from the initiation of radiation therapy till the appearance of disease in irradiated pelvis, detected by imaging and proved histopathologically.

Survival time was calculated from the initiation of the radiation therapy until the last follow-up attendance or death from any cause. Progression-free survival (PFS) was calculated from the initiation of the radiation therapy until the last follow-up attendance without evidence of disease or proof of recurrence. 


\section{Toxicity Assessment}

Acute toxicity is defined as any side effect during and immediately following Brachytherapy up to first follow-up (90 days)

\section{Grading of Reaction}

Grade 1: Mild symptoms of enteritis, proctosigmoiditis, cystitis, etc., that subsides with conservative treatment.

Grade 2: Severe and persistent episodes of proctosigmoiditis, cystitis, etc., that needed hospitalisation but subsided with conservative medical management.

Grade 3: Complications like fistula formation that resulted in surgical manipulation.

Late toxicity is defined as any side effect appearing earliest at 91 days after the end of treatment and are prospectively scored using the Late Effects in Normal Tissues-Subjective, Objective, Management and Analytic score (LENT-SOMA, G0: no side effects, G4: worst side effect, LENT SOMA tables 1995).

\section{Statistical Analysis}

Data was entered in Ms. Excel and analysed using IBM SPSS v22.0. For continuous variables median and interquartile range (I.Q.R.) was used because of skewed data. For the nominal variable, it was expressed in percentages. Statistical significance was defined as $\mathrm{p}$ value $<0.05$.

Survival, the cumulative overall survival (OS) and PFS after radiation therapy, was calculated by the Kaplan-Meier method and Mantel-Cox log-rank test performed for univariate survival curve comparisons. The level of statistical significance was defined as $p$ value $<0.05$.

\section{RESULTS}

735 uterine cervical cancer patients were referred for ICA/VBT after EBRT. Among those, 676 were treated by conventional ICA and 29 by HBT. 30 patients were recruited for this study, as per the inclusion criteria. Table 1 shows the reasons for choosing ISBT. Large volume disease and difficulty in negotiating os to put central tandem were most common indications, but some patients had more than one reason. Table 2 gives patient characteristics like age and stage-wise distribution. Most of the patients were between 41 to $50 \mathrm{yrs}$ and in stage III B disease. Table 3. demonstrates the treatment details. Dose to whole pelvis was $50 \mathrm{~Gy}$. Median fractions (\#) of ISBT were 5 (I.Q.R. 4.75-6). Median volume of HR-CTV measured by CT, in simulation images, was $70 \mathrm{ml}$ ((I.Q.R. $46.4-89.5 \mathrm{ml}$ ). Median BT HR-CTV D90 in EQD2 was 17.5(18.63-28.27) to a planned median dose of 23.6 Gy (1224), while the cumulative median HR-CTV D90 in EQD2 is 73.48 Gy (I.Q.R 71.79 - 73.63 Gy). Median follow-up period for patients still alive at last contact is 12 months (10-16) months. Response assessment is given in Fig. 3. Local control (LC) rate at first follow up (3 months) is 43.3\% (95\% Confidence interval $24.51 \%-62.15 \%$ ) and at $1 \mathrm{yr}$. is $41.38 \%$ (95\% C.I. $22.31 \%-60.45 \%$ ). The median survival time for two-year follow-up was 22.398 months (95\% C.I. 20.26 24.54). The median Progression-free survival time for twoyear follow-up was 19.92 months (95\% C.I. 17.030 - 22.803). Fig. 5 One patient $(3.33 \%)$ had distant metastasis, two patients $(6.67 \%)$ experienced grade 2 rectal bleeding, but no one had bladder complications. At the time of analysis, 13 patients (43.33\%), who were complete responders were alive without disease relapse with (95\% C.I. 25\% - 62\%), 16 $(53.33 \%)$ were alive and had progressive disease, while one patient died because of progression of cervical cancer.

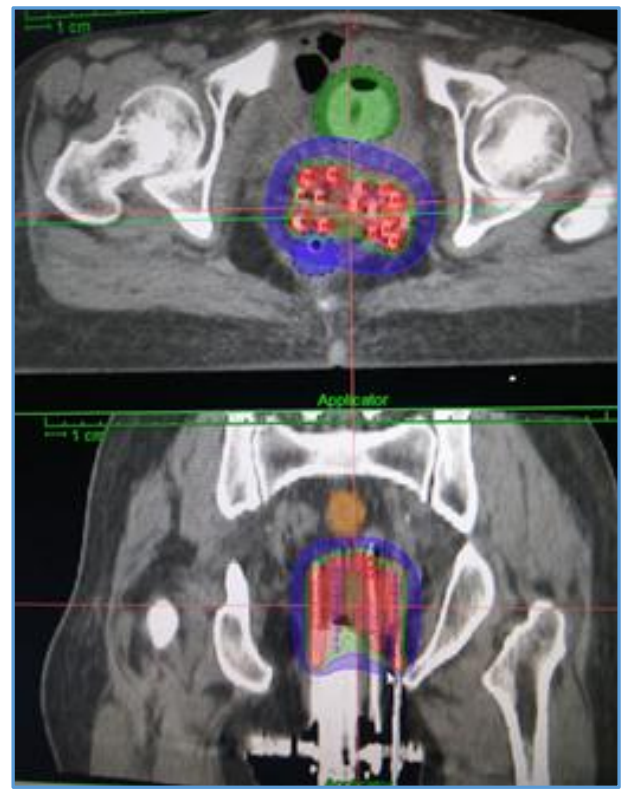

Figure 1. BT Planning

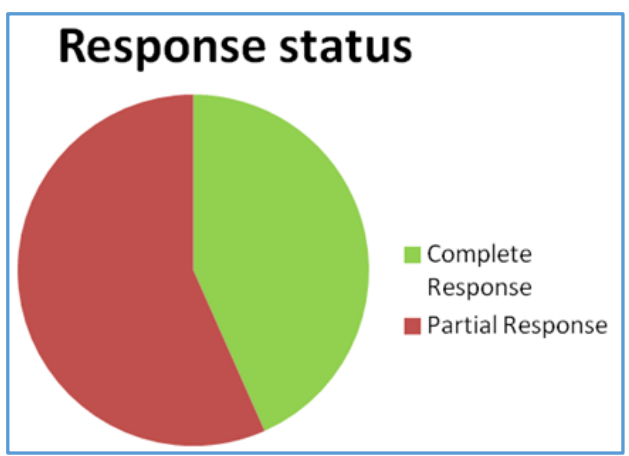

Figure 2. Response Analysis

Mantel-Cox log-rank test gave a chi square value of 0.078 $\mathrm{p}=0.781$.

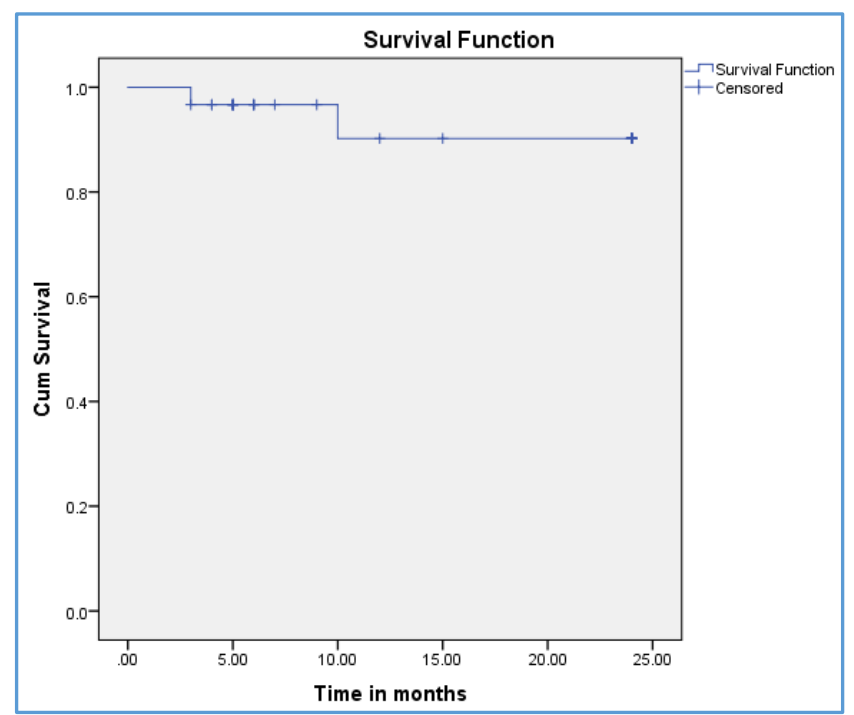

Figure 3. Survival Function 


\begin{tabular}{|c|c|c|}
\hline Reasons & No. of Patients & \% \\
\hline Large Volume (Bulky) & 18 & 60 \\
\hline Os Not Negotiable & 16 & 53 \\
\hline Narrow Vagina & 9 & 30 \\
\hline Vaginal Involvement & 14 & 46 \\
\hline Table 1. Reasons for Selection of Cases for ISBT \\
\hline
\end{tabular}

\begin{tabular}{|c|c|c|}
\hline & Number of Cases & Percentage \\
\hline Age (Years) & 5 & 17 \\
\hline $31-40$ & 13 & 43 \\
\hline $41-50$ & 9 & 30 \\
\hline $51-60$ & & \\
\hline Stage & 8 & 27 \\
\hline Ca CX- IIB & 18 & 60 \\
\hline Ca CX -IIIB & 4 & 13 \\
\hline Post op Vault Rec & Table 2. Age \& Stage-Wise Distribution of Cases \\
\hline \multicolumn{2}{|c|}{}
\end{tabular}

\begin{tabular}{|c|c|c|}
\hline Sl. No. & Features & Median (I.Q.R.) \\
\hline 1. & $\begin{array}{c}\text { Median Dose Prescribed to Whole } \\
\text { Pelvis }\end{array}$ & $50 \mathrm{~Gy}$ \\
\hline 2. & HDR-ISBT Fractions (n) & $5(4.75-6)$ \\
\hline 3. & $\begin{array}{c}\text { Median Number of Needles used in } \\
\text { ISBT }\end{array}$ & $15(11.75-18)$ \\
\hline 4. & Median Volume of HR-CTV (ccm) & $70(46.4-89.5)$ \\
\hline 5. & Median Dose Planned in EQD2 (Gy) & $23.6(12-24)$ \\
\hline 6. & $\begin{array}{c}\text { Median Received Dose of HR-CTV } \\
\text { D90 in EQD2 (Gy) }\end{array}$ & $17.5(18.63-28.27)$ \\
\hline 7. & $\begin{array}{c}\text { Median Cumulative Dose Planned } \\
\text { in EQD2 (Gy) }\end{array}$ & $73.6(71.79-73.63)$ \\
\hline 8. & $\begin{array}{c}\text { Median Cumulative Received Dose } \\
\text { of HR-CTV D90 in EQD2 (Gy) }\end{array}$ & $\begin{array}{c}67.15(62.05- \\
71.22)\end{array}$ \\
\hline 9. & $\begin{array}{c}\text { Median Dose of Bladder D2cc in } \\
\text { EQD2 (Gy) }\end{array}$ & $\begin{array}{c}62.6(60.69-67.31) \\
\text { EQD2 (Gy) }\end{array}$ \\
\hline 10 & $\begin{array}{c}\text { Median Dose of Rectum D2cc in } \\
64.36(61.95- \\
66.19)\end{array}$ \\
\hline 11. & $\begin{array}{c}\text { Median Dose of Sigmoid D2cc in } \\
\text { EQD2 (Gy) }\end{array}$ & $\begin{array}{c}54.83(52.76- \\
56.08)\end{array}$ \\
\hline 12. & Median Follow Up & $\begin{array}{c}12(10-16) \\
\text { months }\end{array}$ \\
\hline & Table 3. Treatment Details \\
\hline
\end{tabular}

\section{DISCUSSION}

Standard treatment for Carcinoma cervix is the combination of EBRT and BT. BT is usually by conventional ICBT, which delivers higher central dose with rapid dose fall-off towards the periphery. Many studies show that most of the patients can be treated by ICBT or HBT. But, there remains a small group of patients, who require, ISBT to achieve better tumour coverage. In our study, $4 \%$ were treated by ISBT. In a similar Study[17] by Naoya Murakami et al., $12 \%$ were treated by ISBT. Our Institution being the only Government Institution, referral centre, practicing ISBT in our state, we can select cases for ISBT.

Median HRCTVD90 in EQD2 is 67.5 Gy Planned dose 73.6(71.79-73.63)\}, which is lower than 87 Gy proposed by Dimopoulas et al. and 80 Gy by ABS Guidelines. HRCTVD90 is lower in our cases because HR-CTV volume is high (more than $80 \mathrm{ccm}$ in $50 \%$ of cases) and to limit dose to OARs, HRCTVD90 has to be lower.

Local control at first follow up is $43.3 \%$ (24.51\% $62.15 \%$ ) compared to other studies from India, $64.7 \%$ as shown by Nandwani et al.,[18] who had given 16-24 Gy in 4-6 \# and Mahant Shetty et al.[19] from Tata memorial hospital, has shown in 37 patients of carcinoma cervix, who underwent inadvertent surgery, overall survival of $64 \%, 10 \%$ patients had Grade III/IV rectal toxicity, $4.5 \%$ had bladder toxicity.

Feder et al.,[20] in 35 patients had shown an LC of $60 \%$ and 9\% complications. Martinez et al.,[21] showed an LC of $83.4 \%$ and $5.1 \%$ complications in 78 patients, while Syed et al. have shown LC of $77 \%$ and complication of $9 \%$ in 60 patients.

The complication rate in our study is only $3 \%$ (1 case of Proctitis) since both median bladder and rectal doses are less than 65 Gy.

Though a prospective study, there remain certain limitations in our study such as not randomized, is from a single institution with a small number of patients. At the end of this study, we have decided to increase the HRCTVD90 with the due limitation of dose to OARs in upcoming patients. Patients are under continued follow up and the results will be presented subsequently.

\section{CONCLUSION}

Treatment by Image-guided ISBT for patients with locally advanced carcinoma cervix in whom the conventional application is not possible, results in good dose-volume histogram (DVH) values and favourable local control with acceptable toxicities is depicted through this prospective single institutional study with a lesser number of patients.

\section{REFERENCES}

[1] Viswanathan AN, Thomadsen B. American Brachytherapy Society Cervical Cancer Recommendations Committee; American Brachytherapy Society. American Brachytherapy Society consensus guidelines for locally advanced carcinoma of the cervix. Part I: general principles. Brachytherapy 2012;11(1):33-46.

[2] Viswanathan AN, Beriwal S, De Los Santos JF, et al. American Brachytherapy Society consensus guidelines for locally advanced carcinoma of the cervix. Part II: high-dose-rate brachytherapy. Brachytherapy 2012;11(1):47-52.

[3] Bethesda M. ICRU report 38. Dose and volume specification for reporting intracavitary therapy in gynecology. ICRU1985;38:1-20.

[4] Tod M, Meredith WJ. Treatment of cancer of the cervix uteri, a revised Manchester method. Br J Radiol 1953;26(305):252-7.

[5] Haie-Meder C, Pötter R, Van Limbergen E, et al. Recommendations from Gynaecological (GYN) GECESTRO Working Group (I): concepts and terms in 3D image based 3D treatment planning in cervix cancer brachytherapy with emphasis on MRI assessment of GTV and CTV. Radiother Oncol 2005;74(3):235-45.

[6] Pötter R, Haie-Meder C, Van Limbergen E, et al. Recommendations from gynaecological (GYN) GEC ESTRO working group (II): concepts and terms in 3D imagebased treatment planning in cervix cancer brachytherapy-3D dose volume parameters and aspects of 3D image-based anatomy, radiation physics, radiobiology. Radiother Oncol 2006;78(1):67-77. 
[7] Dimopoulos JC, Lang S, Kirisits C, et al. Dose-volume histogram parameters and local tumour control in magnetic resonance image-guided cervical cancer brachytherapy. Int J Radiat Oncol Biol Phys 2009;75(1):56-63.

[8] Nag S, Cardenes H, Chang S, et al. Proposed guidelines for image-based intracavitary brachytherapy for cervical carcinoma: report from Image-Guided Brachytherapy Working Group. Int J Radiat Oncol Biol Phys 2004;60(4):1160-72.

[9] Dimopoulos JC, Kirisits C, Petric P, et al. The Vienna applicator for combined intracavitary and interstitial brachytherapy of cervical cancer: clinical feasibility and preliminary results. Int J Radiat Oncol Biol Phys 2006;66(1):83-90.

[10] Jürgenliemk-Schulz IM, Tersteeg RJ, Roesink JM, et al. MRIguided treatment-planning optimization in intracavitary or combined intracavitary/interstitial PDR brachytherapy using tandem ovoid applicators in locally advanced cervical cancer. Radiother Oncol 2009;93(2):322-30.

[11] Tan PW, Koh VY, Tang JI. Outpatient combined intracavitary and interstitial cervical brachytherapy: barriers and solutions to implementation of a successful programme - a single institutional experience. J Contemp Brachytherapy 2015;7(3):25963.

[12] Bailleux C, Falk AT, Chand-Fouche ME, et al. Concomitant cervical and transperineal parametrial high-dose-rate brachytherapy boost for locally advanced cervical cancer. J Contemp Brachytherapy 2016;8(1):23-31.

[13] Martinez A, Cox RS, Edmunson GK. A multiple-site perineal applicator (MUPIT) for treatment of prostatic, anorectal and gynecological malignancies. Int J Radiat Oncol Biol Phys 1984;10(2):297-305.
[14] Dale RG. The application of the linear-quadratic doseeffect equation to fractionated and protracted radiotherapy. Br J Radiol 1985;58(690):515-28.

[15] Bentzen SM, Dörr W, Gahbauer R, et al. Bioeffect modeling and equieffective dose concepts in radiation oncology - terminology, quantities and units. Radiother Oncol 2012;105(2):266-8.

[16] Fowler JF. Sensitivity analysis of parameters in linearquadratic radiobiologic modeling. Int J Radiat Oncol Biol Phys2009;73(5):1532-7.

[17] Murakami N, Kobayashi K, Kato T, et al. The role of interstitial brachytherapy in the management of primary radiation therapy for uterine cervical cancer. J Contemp Brachytherapy 2016;8(5):391-8.

[18] Nandwani PK, VyasRK, Neema JP, et al. Retrospective analysis of role of interstitial brachytherapy using template (MUPIT) in locally advanced gynecological malignancies. J Cancer Res Ther2007;3(2):111-15.

[19] Mahantshetty U, Shrivastava S, Kalyani N, et al. Template-based high-dose-rate interstitial brachytherapy in gynecologic cancers: a single institutional experience. Brachytherapy 2014;13(4):337-42.

[20] Feder BH, Syed AM, Neblett D. Treatment of extensive carcinoma of the cervix with the "transperineal parametrial butterfly": a preliminary report on the revival of Waterman's approach. Int J Radiat Oncol Biol Phys 1978;4:735-42.

[21] Martinez A, Edmundson GK,Cox RS, et al. Combination of EBRT and MUPIT for treatment of locally advanced or recurrent gynecological cancers. Int J Radiat Oncol Biol Phys 1985;11:391-8. 